\title{
Study on Six Degrees of Freedom Arc Welding Robot Trajectory Based on SolidWorks
}

\author{
Zhanqi Liu ${ }^{1, a^{*}}$, Wenming Zhang ${ }^{2, b}$ \\ ${ }^{1}$ Shenyang University, China \\ ${ }^{2}$ Shenyang University, China \\ a18842335807@163.com, b395915920@qq.com
}

\section{Keywords: SolidWorks; Arc welding robot; Saddle shape}

Abstract: Three- dimensional (3D) saddle shape model is built by using SolidWorks software in this paper. Then import the saddle model into a robot off- line programming system. Finally, the simulation study of the SIASUN robot off- line programming system can be used after collecting the saddle model and saving the recognized data type of the robot. Singularities can appear during the period of simulation study. These singularities can disappear after continually adapting the data of saddle model and finally run the predefined program. The robot can be seen moving based on the predefined route within the simulation software interface.

\section{Introduction}

In modern society, with the development of industrialization, control of automation and intelligent have become the trend of development. But most robots in domestic factories mostly using teach programming, and rarely using off-line programming, thus limiting the increase speed in the level of domestic industrial automation. Especially in complex curve design, this paper is going to investigate the saddle shaped curve as an example. This paper would mainly application of SolidWorks software for saddle shaped to establish a three-dimensional (3D) model, and combining SIASUN robot off- line programming system, and do some research on the methods which focus on the complex off- line programming of saddle shaped seam trajectory.

\section{Modelling of Saddle Shape}

Due to its powerful system of Solidworks software and its multiple assemblies, we choose it as a 3D mapping software. The reasons for Selecting Software Solidworks to design 3D saddle trajectory for its simple to application, convenient to operate and easy to learn.

Selection of test parameters: main pipe diameter is $220 \mathrm{~mm}$, length is $380 \mathrm{~mm}$, and the thickness is $5 \mathrm{~mm}$. Branch diameter is $120 \mathrm{~mm}$, length is $190 \mathrm{~mm}$. Open SolidWorks software [1] after selecting the parameters well. The drawing steps are as follows:

Step 1: Create a new file, and click the new sketch icon then, make the XY coordinate as the datum;

Step 2: Click the circle icon command which is used to draw a round and then draw a round with the diameter of $220 \mathrm{~mm}$; click the same command to draw another round with diameter of $219.8 \mathrm{~mm}$. Then click characteristic command, and design the length as $380 \mathrm{~mm}$ while choosing stretch command which is in the characteristic command; click OK;

Step 3: Build a new sketch, the basic plane should based on XY coordinate, then click OK; 
Step 4: Click the circle command and draw out a round with the diameter of $120 \mathrm{~mm}$ again after seizing the center point on the main pipe and click circular command again to draw a round with the diameter of $119.8 \mathrm{~mm}$. Then click characteristic command, and design the length as $300 \mathrm{~mm}$ while choosing stretch command which is in the characteristic command. Its graphics 1 can be seen in the following figure 1;

Step 5: Save files in *.STL format.

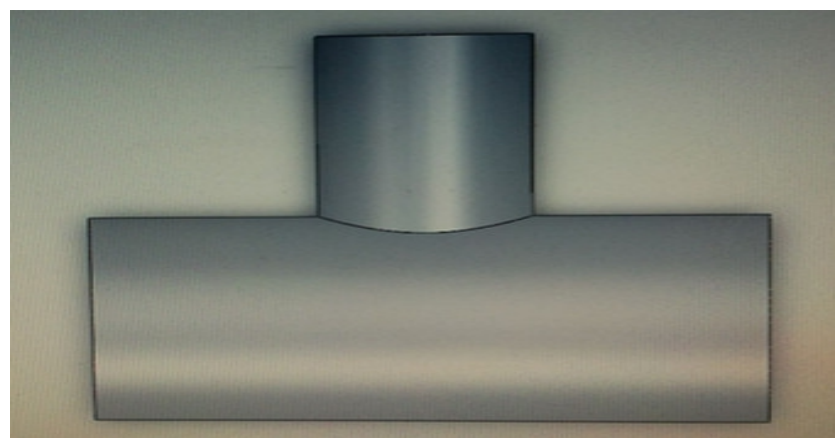

Fig. 1. Diagram of saddle model

\section{Setting of Robot Coordinate System}

\section{Setting of Tool Coordinate System}

A saddle shaped track will be welded this time. First of all, a welding gun should be mounted on the robot wrist. Due to the origin point of the coordinate system of the robot work- piece should be located in the end of welding gun but not on the wrist of the robot, therefore, the tool coordinates system is need to be demarcated, so as to the end effector on the robot can move based on calibration tool coordinate system and can make sure its accuracy and precision. Five points calibration method can be used on the calibration of tool coordinate system. The steps are as follows:

Step 1: Putting the tools on the operating platform and ting them well. Turning on the robot and using the teach box to fix the underlying with the method of five points calibration method;

Step 2: Using the teach box to adjust the robot's posture ${ }^{[2]}$; making the robot go to the standard fixed point and then taking as the first point. Adjusting robot posture again and setting the different point as the second one. Then setting the third point, the fourth point, the fifth point, and then end and exit;

Step 3: Recording the defined tool coordinate system. Its coordinate values are shown in Figure 2.

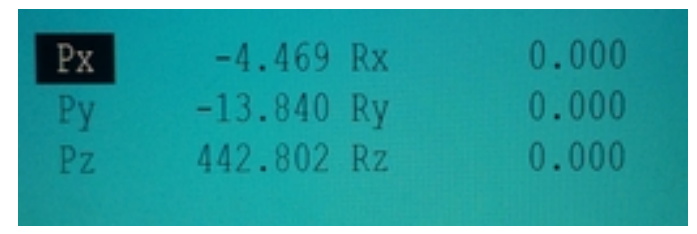

Fig. 2. Chart of tool coordinate value

\section{Setting of Work-piece Coordinate System}

The work-piece coordinate system (also called programming coordinate system) is the coordinate system which is used during programming. This coordinate system is set manually. Establishing the 
work-piece coordinate system is a necessary step before the robot off-line programming. Different methods can be used in different systems. Take the SIASUN robot as an example, the steps of developing the work-piece coordinate system are as follows:

Step 1: Open the design command of the work-piece coordinate system, and select one corner of the working table as the start point of work-piece coordinate system;

Step 2: Apply the teach box to adopt robot reaching the start point and this point should also be located as the origin of the work-piece coordinate system. Select one side of the work-piece as the $\mathrm{X}$ axis, perpendicular to the $\mathrm{X}$ axis as the $\mathrm{Y}$ axis, and $\mathrm{Z}$ axis should be determined by the right hand rule, then exit. The teaching points as shown in Figure 3;

Step 3: Record the well designed work-piece coordinate system, its coordinate values are shown in Figure 4.

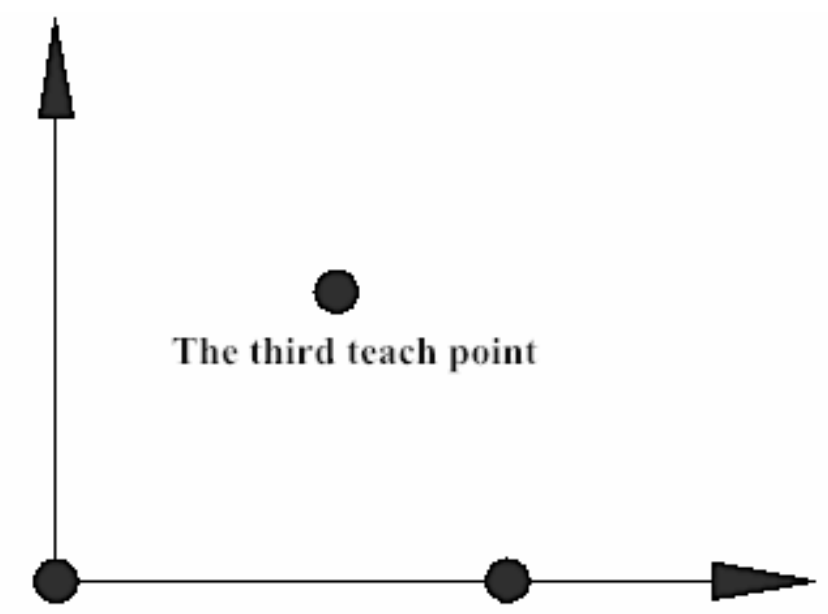

The first teach point The second teach point

Fig. 3. Work-piece Coordinate System Calibration

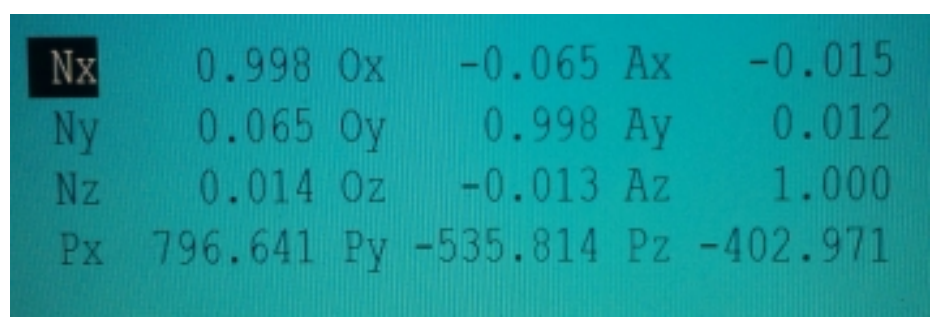

Fig. 4. Chart of Work-piece Coordinate Value

\section{Off-line Simulation in SR_CAM_SOFTWARE (SIASUN Robot Software)}

Simulation is the application of computer on an actual robot system for simulation ${ }^{[3-5]}$. Principle of simulation is by using interactive computer graphics technology and robot theory and forming a robot geometry in computer, then carries on the $3 \mathrm{D}$ display which is used to confirm the changing process of robot and working situation. In this paper, the SR CAM software can be used as an example to simulate the predesigned trajectory. The steps are as follows:

Step 1: Open the software of SR-CAM-SOFTWARE, click the open button and the saddle shaped 3D map which is mentioned above; click processing button; choose the border select button which located in the processing button; recognize the saddle shape trajectory and preservation it for 
$a^{*}$. txt file format;

Step 2: Build a new robot workstation and import the robot model; then set parameters of the above mentioned tool, work-piece coordinate system;

Step 3: Click on and load the core data; and the load the *.txt file into the work space of the robot;

Step 4: Configure track points, and then select all these points for a simulation run. The running status is in line with the actual requirements. The simulation results are shown in figure 5;

Step 5: Save the file at last, which can be used for network remote control application.

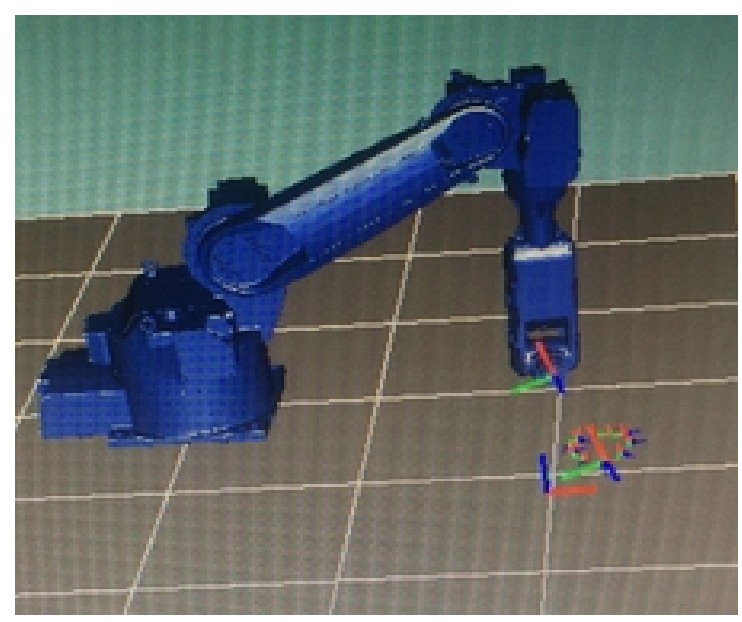

Fig. 5. Chart of Simulation Operation

\section{Conclusions}

The accurate data of saddle model trajectory can be got by drawing the 3D graphics under the help of the SolidWorks software. Such method can improve the accuracy of welding efficiency and its process, but not the teaching- programming.

Programming interface based on SR_CAM_SOFTWARE. Then introduce the well designed trajectory into virtual environment where might appear some singularities. The data of singularity should be revised so as to disappear in the end. Run the well adjust program to complete the whole virtual simulation task.

I gratefully acknowledge the contributions of everyone who help me to finish the paper, especially thanks to the professor Wenming Zhang's guidance and patience!

\section{References}

[1] Xuemei Shang, Yanqing Kang. Equipment Manufacturing Technology, 2013, No. 9: 209- 210, in Chinese.

[2] CSC_V2.3 SIASUN Industrial Robot General Operation Manual [Z].

[3] Users Operation Manual of SR_CAM_SoftWare [Z].

[4] Deyong Wu, Shaolin Liu, Jinxia Wang. Welding Machine, Oct. 2014, Vol. 44, No. 10: 39-43, in Chinese. 
[5] Guanzheng Tan, Xong Xu, Hongfeng Xiao. Journal of Central South University (Science and Technology), Feb. 2005, Vol. 36, No. 1: 103-107. 